\title{
1. Carl Georg Schirren Symposium - 9. Dezember 2006 in Kiel
}

\author{
1st Carl Georg Schirren Symposium - December 9th 2006 in Kiel
}

Bibliografie

DOI 10.1055/s-2007-966305

Akt Dermatol 2007; 33: 100

(C) Georg Thieme Verlag KG .

Stuttgart · New York

ISSN 0340-2541

Korrespondenzadresse

Dr. med. Carl Hermann

Schirren

Dr. Stephan Lischner

Schlossgarten 13

24103 Kiel
Zu diesem Termin hatte die Hautarztpraxis Am Schlossgarten in Kiel im 116. Jahr ihres Bestehens in Memoriam Dr. med. Carl Georg Schirren, 1892 bis 1989, in Zusammenarbeit mit der Akademie für medizinische Fortbildung der Ärztekammer Schleswig-Holstein eingeladen ( $\bullet$ Abb. 1).

Dr. med. Carl Georg Schirren hatte die Praxis selber in zweiter Generation von 1922 bis 1971 geführt. In seinem Grußwort wies der jüngste Sohn und jetzige Betreiber der Praxis auf die große Bedeutung seines Vaters für diese Praxis und für die gesamte Kieler Ärzteschaft in den 50 Jahren seines Tuns hin. In den dann folgenden Vorträgen hatten renommierte Referenten zu diversen Themen innerhalb der Dermatologie berichtet, so Professor Dr. E. Stockfleth aus Berlin über die Bedeutung der HPV-Viren und die moderne Möglichkeit der Vakzinierung.

Er betonte die zentrale Bedeutung der TLRs in der Immunität und wies auf die Bedeutung bestimmter HPV-Typen für die Entstehung des Cervix-Carcinoms hin. Die angesprochene und ausführlich behandelte Möglichkeit einer Vakzinierungsbehandlung könnte zum Schutz vor einer HPV-Erkrankung und folglich vor einem CervixCarcinom geeignet sein.

Dr. Stephan Lischner, der kürzlich in die Praxis Schirren eingetreten ist, behandelte die Therapie epithelialer Hauttumoren, wies auf die Notwendigkeit einer rechtzeitigen Diagnostik und Behandlung hin und stellte entsprechende Beispiele der Behandlung von epithelialen Hauttumoren dar: u.a. Operationen, photodynamische Therapie und die Behandlung mit Cremes als Alternative. H. U. Bock, Kiel, berichtete aus der eigenen dermatologischen Praxis über Fragen der Proktologie, zeigte in einem sehr lebendigen Vortrag, welche Möglichkeiten die entsprechende Diagnostik und Behandlung in einer freien Praxis besitzen.

Prof. Dr. A. Hauschild aus der Universitäts-Hautklinik Kiel berichtete über die Leitlinien der Behandlung des malignen Melanoms im Jahre 2006. Dazu ging er eingehend auf die bisher sehr umstrittenen Sicherheitsabstände ein und verwies darauf, dass eine deutliche Reduzierung derselben bei der Exzision eines malignen Melanoms ein wesentlicher Teil der neuen Leitlinien sei. Die Sentinel-Node-Biopsie wurde dargestellt und auf die Bedeutung als Prognoseparameter für Patienten mit einem malignen Melanom hingewiesen. Abschließend wurde die Bedeutung von Interferon in der adjuvanten Melanom-Therapie behandelt.

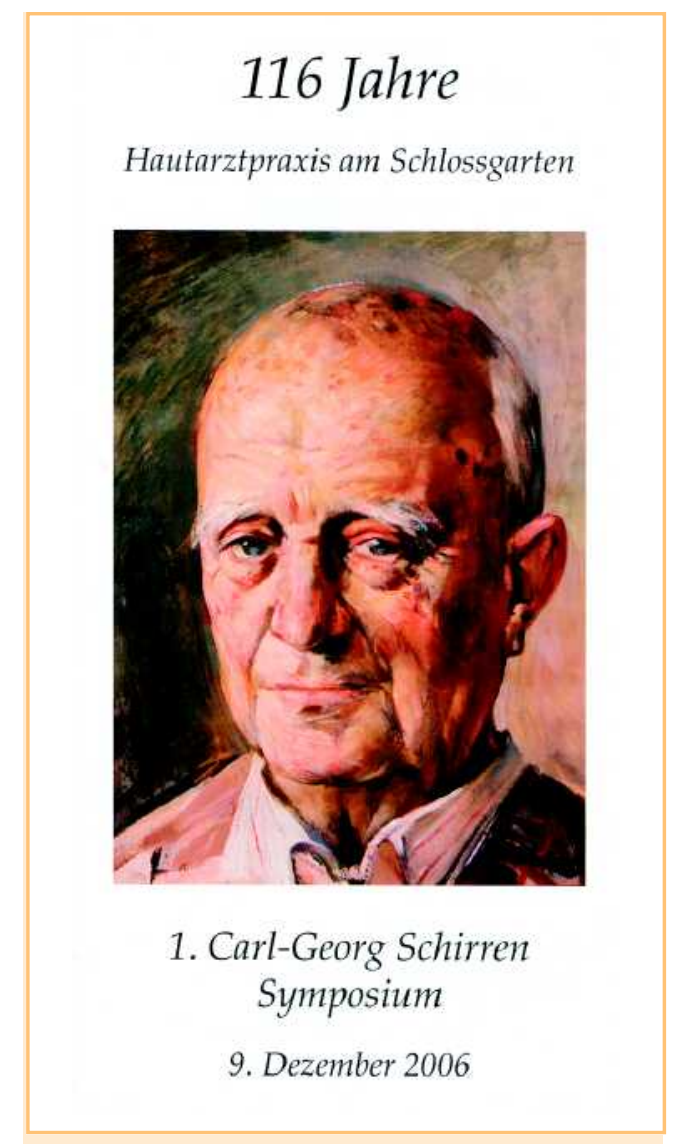

Abb. 1 Einladung

Der Schwiegersohn von Dr. Carl Hermann Schirren, Dr. H.-P. Prieur, Duisburg, berichtete dann über die Bedeutung der Histaminintoleranz, ihre Bedeutung, Diagnostik und Therapie. Das Symposium beendete der Senior der Familie Schirren und der Deutschen Andrologie, Prof. Dr. C. Schirren, Midlum/Föhr, mit dem Thema Andrologie in der Praxis: Möglichkeiten, Grenzen und Ausblick. Dabei wies er auf die andrologischen Daten, die von Carl Georg Schirren in den Jahren 1953-1969 bei 1631 Patienten erfasst worden waren, hin.

Insgesamt nahmen 250 Teilnehmer an diesem 1.Symposium in Kiel teil. Themen, Moderation und äußerer Rahmen dieser Veranstaltung fanden einen großen Zuspruch, so dass die Fortsetzung für den 8. Dezember 2007 geplant ist. Bemerkenswert an diesem Symposium dürfte vor allem sein, dass es sich um eine Veranstaltung von niedergelassenen Dermatologen gehandelt hat. Auch von der Industrie fand diese Veranstaltung mit 15 Ausstellern reichlich Zuspruch. 